\title{
Reflets
}

Revue ontaroise d'intervention sociale et communautaire

\section{Le combat de Catherine}

\section{Pierre}

Volume 7, numéro 1, printemps 2001

Santé mentale et les défis de l’an 2001

URI : https://id.erudit.org/iderudit/026342ar

DOI : https://doi.org/10.7202/026342ar

Aller au sommaire du numéro

Éditeur(s)

Reflets : Revue ontaroise d'intervention sociale et communautaire

ISSN

1203-4576 (imprimé)

1712-8498 (numérique)

Découvrir la revue

Citer cet article

Pierre (2001). Le combat de Catherine. Reflets, 7(1), 161-164.

https://doi.org/10.7202/026342ar

Tous droits réservés (C) Reflets : Revue ontaroise d'intervention sociale et communautaire, 2001

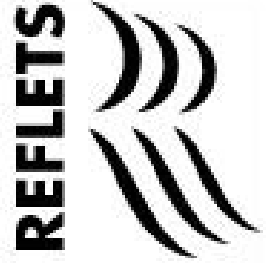

Ce document est protégé par la loi sur le droit d'auteur. L'utilisation des services d'Érudit (y compris la reproduction) est assujettie à sa politique d'utilisation que vous pouvez consulter en ligne.

https://apropos.erudit.org/fr/usagers/politique-dutilisation/ 


\section{Le combat de Catherine}

Pierre, travailleur social, défenseur des malades en santé mentale

J'aimerais partager avec Reflets l'histoire d'une situation récente qui nous présente quelques contradictions au sein du système de santé mentale. Cette situation se déroule dans une petite communauté d'une province canadienne. La province en question refuse qu'on l'identifie et menace Pierre de représailles si l'information est divulguée.

Une femme, que j'appellerai Catherine pour préserver son anonymat, demande d'avoir accès à son dossier médical afin de préparer son audience pour un tribunal. Motif : elle veut se prévaloir de ses droits et contester la décision du psychiatre qui la traite. Plus précisément, elle veut faire appel d'une décision de traitement prise à son sujet. En somme, Catherine possède deux motifs de revendication : s'opposer d'abord au traitement imposé, puis au refus du psychiatre de lui donner accès à son dossier.

Le tribunal constitue un mécanisme d'appel implanté par la Loi sur la santé mentale de la province en question. Depuis qu'il a été institué, une personne peut contester son internement non volontaire ou encore refuser un traitement médical prescrit telle la sismothérapie (électrochocs).

Pour se préparer à cette audience, Catherine demande à consulter son dossier; le psychiatre traitant refuse. L'auteur, qui agit à titre de défenseur des malades, représente Catherine lors de l'audience. À ce moment, Catherine, tout comme le défenseur des malades, peut interroger le psychiatre traitant, appeler des témoins ou même soumettre un mémoire. Les clients peuvent évidemment se faire représenter par un avocat, ce qui n'est pas le cas ici.

Trois membres de la commission de recours écoutent la présentation : un avocat, un psychiatre et un membre du public. 
Ce tribunal est évidemment indépendant de l'administration de tout établissement psychiatrique de la province.

Je vais d'abord présenter un résumé de l'interrogatoire fait par le défenseur des malades auprès du psychiatre traitant pour ensuite émettre quelques commentaires sur les résistances au changement dans le domaine de la psychiatrie.

\section{Résumé de l'interrogation}

Défenseur : Est-il vrai que vous refusez à Catherine, monsieur le psychiatre, l'accès à son dossier médical?

Psychiatre : Oui.

Défenseur : Cette personne présente-elle un danger pour ellemême, oui ou non?

Psychiatre : Non.

Défenseur : Catherine présente-elle un danger pour autrui, oui ou non?

Psychiatre : Non.

Défenseur : Est-ce qu'elle présente un danger pour une tierce personne ou partie, oui ou non?

Psychiatre : Non.

Défenseur : Est-ce qu'elle présente un danger pour le public, oui ou non?

Psychiatre : Non.

Le défenseur poursuit son interrogatoire en se référant à un autre article de la loi.

Défenseur : Monsieur le psychiatre, comment Catherine peut-elle préparer son tribunal prévu par la Loi sur la santé mentale si elle n'a pas accès à son dossier?

Psychiatre: Je ne sais pas! 
Défenseur : Comment ma cliente peut-elle corriger l'information à son dossier si elle n'a pas accès à son dossier médical?

Psychiatre: Je ne sais pas!

Défenseur : Comment ma cliente peut-elle présenter un avis d'amendement si elle n'a pas accès à son dossier médical?

Psychiatre: Je ne sais pas!

Défenseur : Docteur, comment Catherine peut-elle effectuer un avis de désaccord si elle n'a pas accès à son dossier médical?

Psychiatre: Je ne sais pas!

Défenseur : Docteur, l'accès à son dossier médical présente-il un danger à son traitement, oui ou non?

Psychiatre : Non.

Le défenseur des malades résume son plaidoyer et insiste sur le fait que la cliente est compétente et que ses droits sont lésés.

\section{Décision}

Après délibération, les membres de la commission de recours accordent à Catherine accès à son dossier médical.

Des décisions judiciaires telles Reid c. Gallagher, Schulman c. Mallet, Joshua Walker c. le Ministère ainsi que la Charte canadienne des droits et libertés reconnaissent la validité d'une décision du genre.

\section{Commentaires}

Dans cette affaire, Catherine a évidemment gagné son appel. Cette forme d'empowerment constitue beaucoup plus qu'une procédure légale; la portée de ces contestations judiciaires et administratives illustre les changements de mentalités à l'égard des personnes aux prises avec des problèmes psychiatriques vivant en institution ou 
des ex-psychiatrisés vivant dans la communauté. Les personnes ayant un problème de santé mentale ne sont plus isolées, victimisées, exclues de la vie en société. Elles peuvent retenir les services d'un avocat et profiter de la protection prévue par la Charte canadienne des droits et libertés. Nombreuses sont les personnes en milieu psychiatrique qui ont exercé leur droit grâce à cette nouvelle loi.

Certes, la pharmacothérapie a fait des pas de géant en psychiatrie. Les diverses thérapies psychosociales permettent, dans certains cas du moins, un meilleur fonctionnement de la personne en communauté. Une politique de logement, lorsqu'elle est bien rodée, facilite la réinsertion sociale. Les personnes aux prises avec un problème de santé mentale ne sont plus considérées comme possédées du démon, folles ou stupides. Elles peuvent se prévaloir de leurs droits. Des mécanismes plus rigoureux sont mis sur pied pour les personnes psychiatrisées aux prises avec des conflits avec le code criminel du Canada. Et même là, il existe un mécanisme de recours, la Commission d'examen, ou l'aide juridique qui leur permet de profiter des services d'un avocat, pour une justice plus équitable.

L'advocacy pratiquée dans un tel cas favorise la protection des personnes démunies et l'expression des intérêts des clients en plus de corriger des lacunes et des injustices dans les services des ministères responsables de la santé mentale en rapport à l'internement non volontaire et au traitement médical non désiré. Une situation comme celle de Catherine continuerait de se produire régulièrement s'il n'y avait pas droit de recours.

Au cours de mes nombreuses années passées au Service de défense des malades, je me suis demandé si l'advocacy ne pouvait pas devenir encore plus communautaire qu'administrative et judiciaire. Et plus collective que gouvernementale. Des recherches axées sur les données statistiques du service de défense des malades et sur l'enjeu de certains dossiers-clés permettraient au public de mieux saisir la valeur historique et légale des revendications sociopolitiques des bénéficiaires en santé mentale. L'intervention structurelle et conscientisante faciliterait des alliances collectives fructueuses tant sur le terrain qu'en recherche-action, pour un plus grand respect des droits des gens opprimés par leurs milieux, mais aussi, comme dans le cas de Catherine, par des professionnels. 